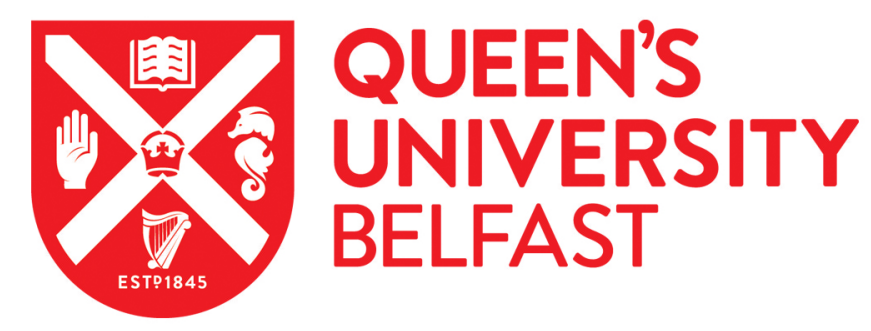

\title{
Enhancing the security of FinTech applications with map-based graphical password authentication
}

Meng, W., Zhu, L., Li, W., Han, J., \& Li, Y. (2019). Enhancing the security of FinTech applications with mapbased graphical password authentication. Future Generation Computing Systems, 101, 1018-1027.

Published in:

Future Generation Computing Systems

Document Version:

Peer reviewed version

Queen's University Belfast - Research Portal:

Link to publication record in Queen's University Belfast Research Portal

Publisher rights

Copyright 2019 Elsevier Ltd

This manuscript is distributed under a Creative Commons Attribution-NonCommercial-NoDerivs License

(https://creativecommons.org/licenses/by-nc-nd/4.0/), which permits distribution and reproduction for non-commercial purposes, provided the author and source are cited.

\section{General rights}

Copyright for the publications made accessible via the Queen's University Belfast Research Portal is retained by the author(s) and / or other copyright owners and it is a condition of accessing these publications that users recognise and abide by the legal requirements associated with these rights.

Take down policy

The Research Portal is Queen's institutional repository that provides access to Queen's research output. Every effort has been made to ensure that content in the Research Portal does not infringe any person's rights, or applicable UK laws. If you discover content in the Research Portal that you believe breaches copyright or violates any law, please contact openaccess@qub.ac.uk. 


\title{
Enhancing the Security of FinTech Applications with Map-Based Graphical Password Authentication ${ }^{\text {负 }}$
}

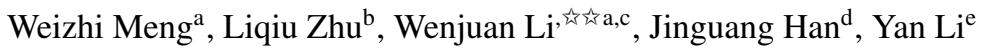 \\ ${ }^{a}$ Department of Applied Mathematics and Computer Science, Technical University of Denmark, Denmark \\ ${ }^{b}$ FinTech Startup, Macau, China \\ ${ }^{c}$ Department of Computer Science, City University of Hong Kong, Hong Kong SAR \\ ${ }^{d}$ School of Electronics, Electrical Engineering and Computer Science, Queen's University Belfast, UK \\ ${ }^{e}$ School of Information Systems, Singapore Management University, Singapore \\ ${ }^{f}$ E-mail address:weme@dtu.dk,wenjuan.li@my.cityu.edu.hk
}

\begin{abstract}
With the rapid development of information technology (IT) in financial industry, financial technology (FinTech) has become an emerging area for enterprises and organizations. Due to the wide adoption of IT, various FinTech applications are used by financial industry to help process information and offer financial services. Traditionally, textual passwords are the most widely deployed authentication mechanism, while having many known limitations. As a result, there is a need to enhance the security of FinTech authentication against cyber-criminals. As an alternative, graphical passwords (GPs) are considered as one promising solution to complement traditional password-based systems. In the literature, various GP schemes were proposed such as PassPoints, DAS, Cued Click Points, GeoPass, etc. In this work, we identify that multiple password inference has become a challenge for most GP schemes, and thus design RouteMap, a map- and route-based GP to further improve the security of FinTech applications. This scheme requires users to create a route on a world map as their credentials. In the evaluation, we involved a total of 120 participants, among which 60 of them have financial (FinTech) background, and investigated the performance of RouteMap by comparing it with two similar schemes. Our results demonstrate that participants can achieve better performance using RouteMap in the aspects of authentication accuracy and multiple password memory. Our effort attempts to complement existing studies and stimulate more research on the combination of GP and FinTech.
\end{abstract}

Keywords: FinTech Application, User Authentication, Multiple Password Inference, Graphical Passwords, Map Passwords, Security and Usability.

\section{Introduction}

The fast growth of information technology (IT) has provided a profound impact on the traditional financial sector. Financial technology (FinTech) is considered as a distinguishing taxonomy that describes the financial techniques in a wide range of operations for many enterprises and organizations, which mainly aims to improve the service quality by means of various IT applications [11]. According to the data collected from the PwC's DeNovo platform, the funding of FinTech startups has increased at a compound annual growth rate (CAGR) of $41 \%$ over the last four years, with over US\$40 billion in cumulative investment [13]. Cutting-edge FinTech companies and financial innovation are changing the competitive landscape and up to $82 \%$ incumbents expect to increase FinTech partnerships in the next three to five years.

With the rapid development in FinTech, various FinTech applications have been developed to help process information and

\footnotetext{
A preliminary version of this paper appears in the proceedings of the 9th International Conference on Network and System Security (NSS), pp. 147-161, 2015 [24].

Corresponding author: Wenjuan Li with Email address: wenjuan.li@my.cityu.edu.hk
}

offer financial services. However, security is one of the major concerns for most financial firms as traditional IT may bring the same vulnerabilities to FinTech applications. For example, cyber-criminals can access the financial devices and steal sensitive information. In this case, there is a significant need to develop appropriate mechanisms to enhance the process of verifying current application users [8].

In the past decades, textual passwords should be the most commonly used authentication method, where users have to input correct textual strings for registration and authentication. However, it has long been recognized that traditional text-based passwords may suffer from many issues associated with both security and usability aspect [43, 44]. For instance, users are hard to remember their passwords, especially complex and random passwords for a long time, which is known as long-term memory (LTM) limitation. Instead, users are likely to choose simple passwords, which would significantly degrade the security of authentication. The recent study shows that this situation would be even worse than previously believed (i.e., there is little variation in guessing difficulty) [1].

To complement textual password authentication, graphical passwords (GPs) have been developed as a promising alternative for user authentication. It is identified that humans general- 
ly have better memory and recognition capabilities for images than textual strings $[32,34]$. This observation has motivated the design of many graphical password schemes, which mainly involve recognizing images or reproducing a drawing on images. For example, Jermyn et al. [15] designed $D A S$, a graphical password that allowed users to draw their own passwords on a 2D grid. Wiedenbeck et al. [42] proposed PassPoints, a system that allowed users to click on any place on an image in creating their passwords. Chiasson et al. [2] proposed a click-based graphical password scheme called Cued Click Points (CCP), which requires users to choose one click-point per image for a sequence of images. The next image displayed is based on the previous click-point, hence users could receive immediate implicit feedback and decide whether they are on the correct path.

Motivations. In practice, it is very common that people create more than one password for different accounts. For financial people, they may have to manage different passwords for various FinTech applications that are used to process distinct data or fulfill special financial requirements (e.g., security level). However, remembering a password for one application might affect users' memory of other passwords, which is known as multiple password interference. This situation often happens when users have to remember passwords for multiple systems where the memories of different passwords may interfere with each other $[4,41]$. To reduce the burden of remembering multiple passwords, a good authentication scheme should be easy for users to remember multiple credentials. While similar to textual passwords, we notice that multiple password memory has also become an issue for most GP schemes, in which users are not easy to remember all created graphical patterns after a time period. In this work, our attempt is to develop an appropriate GP scheme to help lighten the LTM limitation and multiple password interference.

Contributions. To achieve our goals, we set two major targets: the first one is to investigate whether users have inference issue of remembering multiple graphical passwords; and the second one is to design a proper GP scheme for better multiple password memory. For such purpose, we performed a set of user studies accordingly. The first two studies with general and FinTech participants aim to evaluate the multiple password inference of two popular GP schemes: DAS and PassPoints. Then we design a map- and route-based graphical password scheme called RouteMap, which allows users to draw a route on a map as their passwords. We then conducted two studies with general and FinTech participants to investigate the performance of RouteMap, as compared with two other similar schemes. Our contributions can be summarized as follows.

- We begin by conducting a user study to explore whether users are capable of remembering multiple graphical passwords like DAS and PassPoints. These two schemes are selected due to their popularity and simplicity. It is found that these GP schemes have the issue of multiple password memory inference, and there is a need for designing a more usable GP scheme.

- Then, we introduce RouteMap, which is a map- and routebased authentication scheme, allowing users to draw a route on a world map as their credentials. The creation of a route is believed to provide hints for users to remember their multiple passwords in a usable way. We also provide several guidelines and rules for users on how to create a route for better memory.

- To investigate its performance, our evaluation with a set of studies involved 60 general participants, and 60 FinTech participants who have financial background. Experimental results indicate that participants can achieve better performance using RouteMap in the aspects of both multiple password memory and long-term memory, as compared with two similar map-based schemes.

Roadmap. The remaining parts of this paper are organized as follows. In Section 2, we introduce related research studies regarding graphical passwords, especially map-based GPs. Section 3 describes our study to investigate the issue of multiple password inference for DAS and PassPoints. Section 4 introduces our designed scheme of RouteMap in detail and evaluates its performance as compared with two similar schemes. Finally, we make a discussion in Section 5 and conclude our work in Section 6.

\section{Related Work}

In this section, we focus on introducing the background of graphical passwords, including the typical classification and map-based schemes.

\subsection{GP Classification}

Graphical password schemes can be classified into three folders [3, 37]: recognition-based scheme (i.e., recognizing images), pure recall-based scheme (i.e., reproducing a drawing without a hint) and cued recall-based scheme (i.e., reproducing a drawing with hints).

- Recognition-based GPs. The recognition-based schemes require users to select one or more images from a large set. For instance, the application of PassFaces [33] requires users to recognize a set of human faces during authentication. The scheme of Story [6] requires users to recognize a set of images like people and food from a large image pool, and then to remember all the selected images in a story-sequence, aiming to enhance the memory.

- Pure recall-based GPs. This kind of GP usually requests users to draw something on an image as their passwords. A typical example of these GPs is DAS [15], which requires users to draw on a grid. Similarly, the scheme of PassGo [39] requests users to select intersections on a grid as a way to input a password. Based on Pass-Go, Android unlock patterns have been developed on Android phones, which are a tuned application requiring users to unlock their phones by inputting correct patterns. ${ }^{1}$ Several similar schemes can be referred to $[9,18]$.

\footnotetext{
${ }^{1}$ https://www. berkeleychurchill.com/software/ android-pwgen/pwgen.php.
} 
- Cued recall-based GPs. This kind of GP demands users to click on a sequence of points to construct their secrets. The system of PassPoints belongs to this category where users have to recall a sequence of five selected points. As another example, Chiasson et al. [5] designed Persuasive Cued Click-Points (PCCP), which requires users to click a point on each of a sequence of background images.

Katsini et al. [16] presented some preliminary results on the graphical password composition, among users with different cognitive strategies. The existing GP schemes are mainly based on users' actions like choice, click and draw. To further enlarge the password space, various hybrid GP schemes are also proposed in the literature, like click-draw based GP scheme [20] that combined all interactions in generating a password, including clicking, selecting and drawing. Meng and Liu [30] proposed TMGMap, a touch movement-based graphical password scheme, in which users can draw their secrets on a world map via touch movement events on smartphones. Meng et al. [31] introduced CPMap, a click-points map-based GP scheme that allows users to choose one place on a world map at first and then choose a point or an object on an image associated with the previously selected location. Some other relevant studies on improving the performance of GPs can be referred but not limited to [7, 14, 21, 22, 23, 25, 27, 28, 45].

\subsection{Map-based Graphical Passwords}

The initial idea of using digital map as a graphical password first appeared in [10], but their work failed to provide much details. Then, Spitzer et al. [36] developed a graphical approach by combining user's familiarity with navigating through Google maps. In their work, users were presented with an image of the United States and they should simply click at where the key destination is located, using an approach of zooming levels. Their results with over 50 participants indicated that up to $60 \%$ participants rated their system as easier to remember than text in terms of memorability.

Then, more map-based graphical passwords were designed from 2012. Georgakakis et al. [12] proposed a GP scheme called NAVI, where the credentials of a user are the username and a relevant password formulated by drawing a route on a predefined map. They provided an analysis regarding the password strength, but did not provide any user study. Sun et al. [38] proposed a map-based GP authentication system called PassMap, in which a password consists of a sequence of 2 click-points selected on a world map. Their user study showed that PassMap passwords are easy to remember in practice. Thorpe et al. [40] designed GeoPass, a digital map-based authentication scheme, where a user chooses a place as his or her password. In the user study, they found that $97 \%$ participants were able to remember their location password over the span of 8-9 days and most without any failed login attempts. It is worth noting that PassMap and GeoPass are very similar in that secrets are constructed by clicking one or two places on a world map (e.g., Google map). Then, MacRae et al. [19] proposed GeoPassNotes, asking users to further select a note associated with their chosen location in the second step. Shin et al. [35] then implemented a modified version of GeoPass on a mobile device.

In practice, users have a need to handle more than one accounts, i.e., users may generate different passwords for logging into each FinTech application. However, remembering a password for one application might affect users' memory of other passwords, which is known as multiple password interference [41]. Meng et al. [26] introduced a study of recalling multiple passwords between text passwords and touch screen unlock patterns and found that participants in the unlock pattern condition with three accounts can outperform those in the text password condition. Further, Meng et al. [29] conducted a study with 60 participants to investigate the recall of multiple passwords between text passwords and map-based passwords under various account scenarios. In particular, each participant has to create six distinct passwords for different account scenarios. It is found that participants in the map-based graphical password scheme could perform better than the textual password scheme in both short-term (one-hour session) and long term (after two weeks) password memorability tests. In [24], we designed a scheme called RouteMap, allowing users to draw a route on a world map as their secrets. In this work, we extend the idea of RouteMap and investigate its performance to the field of FinTech.

\section{Multiple Graphical Password Inference}

In this section, we conducted a user study with 50 participants to investigate the inference issue of multiple password memory on GPs. We selected two GP schemes: DAS and PassPoints, due to their popularity and simplicity. The former is a pure recall-based GP, where users can draw their secrets on a grid. The latter is a cued recall-based GP, where users have to remember a sequence of several clicks. All participants are volunteers and have no background on information security (i.e., no participant has taken any courses related to information security before). Table 1 describes the detailed information of participants. Further, we conducted another user study with 20 FinTech participants to validate our results.

Methodology. Both GP schemes were implemented under the same computer settings and we introduced our objectives to all participants. Two password patterns from these systems are depicted in Figure 1 (a) and Figure 1 (b). More scheme details can be referred to $[15,42]$. To avoid bias, we set a file including all steps in the lab study and gave a detailed description to all participants based on the same steps (i.e., how to use these two example systems).

Before the start of evaluation, each participant had 3 trials to get familiar with the example systems. In this study, we require all participants to create 5 passwords for each scheme, in which each password corresponds to a scenario as follows: the first password is created for an email account (personal use), the second one is created for a bank account, the third one is created for another email account (commercial use), the fourth one is created for a forum account and the last one is created for a social networking account. The detailed steps in each experiment are shown as below: 
Table 1: Detailed information of participants in the user study.

\begin{tabular}{|l|c|c|l|c|c|}
\hline Age Range & Male & Female & Occupation & Male & Female \\
\hline \hline $18-25$ & 8 & 9 & Senior people & 3 & 2 \\
\hline $25-35$ & 8 & 8 & Students & 16 & 12 \\
\hline $35-45$ & 4 & 3 & Researchers & 3 & 3 \\
\hline $45-55$ & 2 & 3 & Engineers & 3 & 3 \\
\hline $55-60$ & 3 & 2 & Business people & 2 & 3 \\
\hline
\end{tabular}

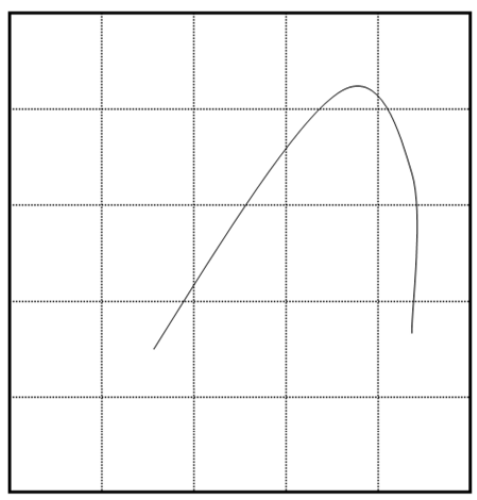

(a) DAS

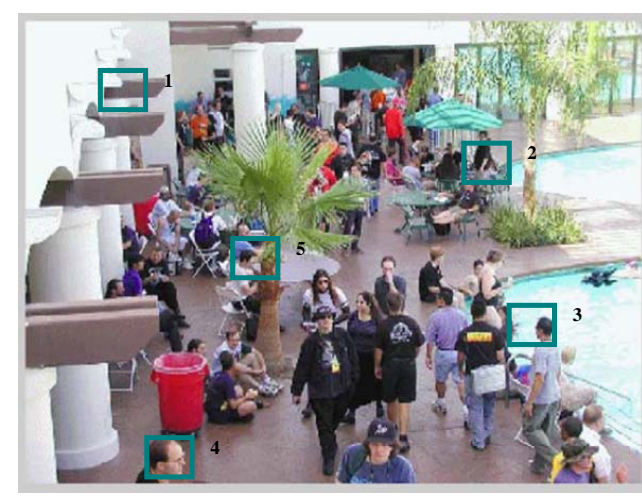

(b) PassPoints

Figure 1: Two graphical password schemes: (a) DAS and (b) PassPoints.

- Experiment1. This experiment requires each participant to create $5 D A S$ passwords.

- Step 1. Creation: creating a password following the rules of $D A S$.

- Step 2. Confirmation: confirming the password by drawing the same secrets in the correct place. If users incorrectly confirm their password, they can retry the confirmation or return to Step 1.

- Step 3. Login: logging into the system with the created passwords. Users can cancel an attempt if they notice an error.

- Step 4. Feedback: All participants are required to complete a feedback form about the password creation and confirmation.

- Experiment2. This experiment requires each participant to create 5 PassPoints passwords.

- Step 1. Creation: creating a password following the rules of PassPoints.

- Step 2. Confirmation: confirming the password by drawing the same secrets in the correct place. If users incorrectly confirm their password, they can retry the confirmation or return to Step 1.

- Step 3. Login: logging into the example system with the created passwords. Users can cancel an attempted login if they notice an error.

- Step 4. Feedback: All participants are required to complete a feedback form about the password creation and confirmation.
Each participant had to finish these two experiments in the same day. After three weeks, we require all participants to return and input all created passwords for these two schemes. Afterwards, we provided another feedback form for participants about their password creation and memory.

Result analysis. Our main purpose of this study is to explore whether users are able to remember and manage multiple graphical passwords. That is why we mainly describe and analyze users' performance after three weeks. The success rates of login to DAS and PassPoints within three attempts are described in Table 2. Three trials are determined based on the observation that most host \& network accounts only allow an authentication error within three times. We have several major observations as below:

- Overall performance. It is found that participants could only achieve a success rate of $52.4 \%$ and $45.2 \%$ for DAS and PassPoints at the first attempt, respectively. After three trials, the success rate could be increased to $70.4 \%$ and $66.8 \%$, respectively.

- Age impact. In Table 2, we also present the results according to three groups with different age-ranges. It is noticed that participants who are aged from 35 to 45 could achieve the best performance in the study. That is, younger participants could have some advantages in multiple password memory. However, the success rate for both schemes was less than $80 \%$ after three attempts (where the rate is $77.1 \%$ for DAS and $71.4 \%$ for PassPoints).

Based on the results, it is found that participants did not show satisfied capability in remembering these two GP schemes. To 
Table 2: Success rate for login to DAS and PassPoints after three weeks.

\begin{tabular}{|l|c|l|c|}
\hline Experiment (DAS) & Successful Login & Experiment2 (PassPoints) & Successful Login \\
\hline 1st time & $132 / 250(52.4 \%)$ & 1st time & $123 / 250(45.2 \%)$ \\
\hline 2nd time & $163 / 250(65.2 \%)$ & 2nd time & $150 / 250(60.0 \%)$ \\
\hline 3rd time & $176 / 250(70.4 \%)$ & 3rd time & $167 / 250(66.8 \%)$ \\
\hline \hline$D A S($ Age in $[18,35])$ & Successful Login & PassPoints (Age in [18, 35]) & Successful Login \\
\hline 1st time & $98 / 165(59.4 \%)$ & 1st time & $80 / 165(48.5 \%)$ \\
\hline 2nd time & $108 / 165(65.5 \%)$ & 2nd time & $105 / 165(63.6 \%)$ \\
\hline 3rd time & $115 / 165(69.7 \%)$ & 3rd time & $114 / 165(69.1 \%)$ \\
\hline \hline DAS (Age in [35, 45]) & Successful Login & PassPoints (Age in [35, 45]) & Successful Login \\
\hline 1st time & $12 / 35(34.3 \%)$ & 1st time & $13 / 35(37.1 \%)$ \\
\hline 2nd time & $22 / 35(62.9 \%)$ & 2nd time & $20 / 35(57.1 \%)$ \\
\hline 3rd time & $27 / 35(77.1 \%)$ & 3rd time & $25 / 35(71.4 \%)$ \\
\hline \hline DAS (Age in $[45,60])$ & Successful Login & PassPoints (Age in $[45,60])$ & Successful Login \\
\hline 1st time & $21 / 50(42.0 \%)$ & 1st time & $20 / 50(40.0 \%)$ \\
\hline 2nd time & $33 / 50(66.0 \%)$ & 2nd time & $25 / 50(50.0 \%)$ \\
\hline 3rd time & $34 / 50(68.0 \%)$ & 3rd time & $28 / 50(56.0 \%)$ \\
\hline
\end{tabular}

Table 3: The main questions and relevant scores for both common and FinTech participants.

\begin{tabular}{|l|c|c|}
\hline Questions / Score (average) & 50 Participants & 20 FinTech Participants \\
\hline \hline 1. I could easily remember DAS passwords after one month & 4.5 & 4.3 \\
\hline 2. I could easily remember PassPoints passwords after one month & 4.2 & 4.1 \\
\hline 3. Are you willing to use DAS passwords in practice & 3.2 & 3.5 \\
\hline 4. Are you willing to use PassPoints passwords in practice & 4.7 & 4.1 \\
\hline 5. I can manage multiple DAS passwords & 3.5 & 3.1 \\
\hline 6. I can manage multiple PassPoints passwords & 4.3 & 3.8 \\
\hline
\end{tabular}

investigate this issue, we collect the feedback forms and present some key questions with relevant scores in Table 3. Tenpoint Likert scales were used in each feedback question, where 1 -score indicates strong disagreement and 10-score indicates strong agreement.

It is observed that participants could not remember these $\mathrm{t}$ wo GPs for a long time, where the average scores of the first and the second question were lower than 5. In addition, most participants were not willing to use these GPs in real-world applications. Similarly, most participants stated that it is difficult to remember multiple GPs. We informally interviewed most participants and identified two major reasons: (1) for $D A S$, it is not easy to link the graphical password to the corresponding accounts; and (2) for PassPoints, it is easily to forget the click-points when creating more than 3 passwords. Up to $80 \%$ participants reported that they have more than 5 different textual passwords in use.

FinTech participants. To validate the observations, we further conducted a study with 20 participants who are doing financial work or had a financial job before. All participants have no background on information security and would receive a 50\$ coupon after the study. The study followed the same steps as above and the results are shown in Table 4.

It is found that FinTech participants could reach a success rate of $45 \%$ and $48 \%$ for each DAS and PassPoints at the first attempt, while the success rate could be finally increased to $72 \%$ and $68 \%$, respectively. We used Chi-square $\left(\chi^{2}\right)$ tests to help analyze non-ordered categorical or nominal data (e.g., success rates), where a value of $\rho<0.05$ indicates that the results are statistically significant between two conditions. As compared with the results in Table 2, we found there is no statistically significant difference. In addition, Table 3 also shows the feedback from the FinTech participants, and similarly, the results have no statistically significant difference.

Discussion. The results demonstrate that the inference of multiple password memory has become a challenge for both common and FinTech users when using graphical passwords in practice. Subsequently, we argue that this issue should be given more attention when designing a GP scheme. There is a great need for developing suitable GP schemes for better multiple password memory. In the study, we also have two observations: (1) a background image can help users to remember their secrets, and (2) users could be provided with a few guidelines for creating their GPs.

\section{RouteMap Design}

In this section, we describe our proposed RouteMap in detail and then present a new user study to investigate its performance, as compared with two similar schemes.

\subsection{RouteMap Scheme}

The proposed scheme of RouteMap is a kind of map-based graphical password, which requires users to draw a route on a map (e.g., Google map). For better password memory, we advocate the use of a world map due to the following three merits. 
Table 4: FinTech participants: success rate for login to DAS and PassPoints after three weeks.

\begin{tabular}{|l|c|l|c|}
\hline Experiment $A(\mathrm{DAS})$ & Successful Login & Experiment $B$ (PassPoints) & Successful Login \\
\hline 1st time & $45 / 100(45 \%)$ & 1st time & $48 / 100(48 \%)$ \\
\hline 2nd time & $61 / 100(61 \%)$ & 2nd time & $63 / 100(63 \%)$ \\
\hline 3rd time & $72 / 100(72 \%)$ & 3rd time & $68 / 100(68 \%)$ \\
\hline
\end{tabular}

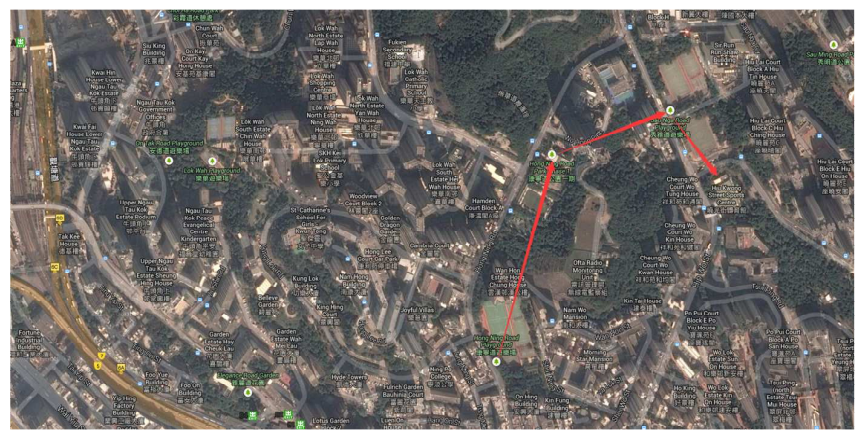

(a) RouteMap with sight

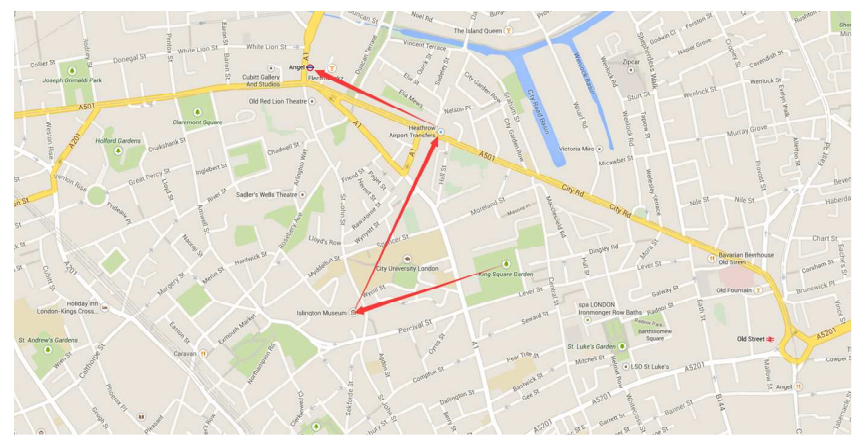

(b) RouteMap without sight

Figure 2: Two examples of RouteMap passwords: (a) a pattern with sight and (b) a pattern without sight.

- Map-based graphical passwords such as PassMap and GeoPass can provide a large password space (e.g., $2^{36.9}$ for GeoPass [40] under unknown adversary).

- A world map can be easily zoom-in or zoom-out, hence users can freely choose a satisfied background image.

- Previous studies had shown that map-based GPs could provide good usability (i.e., GeoPass study [40] shows that 97\% participants can remember their passwords over a span of 8-9 days).

Our scheme. To enhance the memory, RouteMap allows users to create a road on a world map as their credentials, with or without sight. This is because people may have their own preferred background images. In Figure 2, we present two examples of RouteMap patterns with and without sight.

Taking the pattern in Figure 2 (b) as an example, the user needs to click on the playground first, then move and click on a park and another playground, and finally click and stop at the sport center. In this case, a RouteMap pattern includes sight information, first click-point and the whole route. In particular,

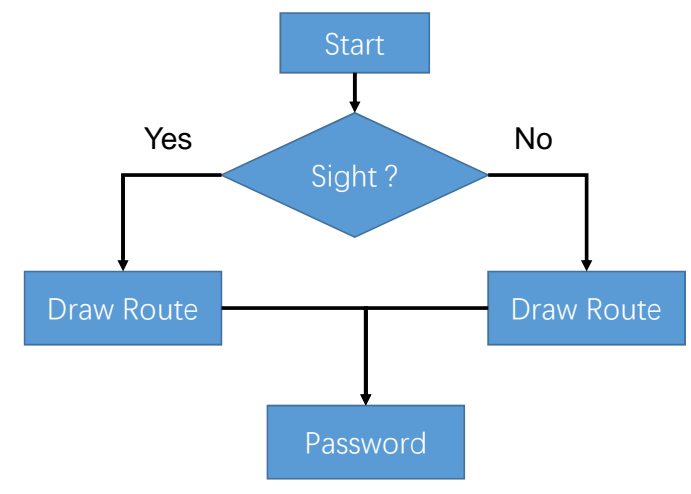

Figure 3: Workflow of password generation.

RouteMap is different from other similar map-based schemes in the following aspects.

- RouteMap allows users to choose whether to use sight (a satellite background) or not, aiming to improve users' memory by placing them in a preferred environment. This selection will be included in the final pattern stored in the system.

- RouteMap only allows users to draw straight lines between different locations. This aims to improve the usability, as it is noted that drawing curves is not easy for users with a mouse input device (i.e., it may consume much more time and increase error rates).

- RouteMap provides a simple guideline for users, which recommends users to create a route based on their own memory such as previous tours and visits. It is found that tour-route or visit-route pattern is private for users, which may enhance the memory of various clicks on a map.

Implementation of RouteMap. The workflow of password generation is depicted in Figure 3. We built a prototype system of RouteMap in our lab environment, which is similar to the design of PassMap and GeoPass. To fetch a real world map, we utilized Java scripts and Google Maps API, and our system can provide move (drag), zoom in, zoom out, and search functions. When users zoom-in or zoom-out the map, RouteMap could report the zoom levels. The search function can facilitate users to shift to a specific area quickly, and the use of zoom-in and zoom-out can help locate a proper area smoothly. Then, users can create a password by clicking at a location and moving the mouse to the next places. Based on the prior studies [21, 38, 40 ], we set the error tolerance to a $21 \times 21$ pixel box around the place where the users clicked. It is worth noting that the error 
Table 5: Detailed information of participants in the user study with general participants.

\begin{tabular}{|l|c|c|l|c|c|}
\hline Age Range & Male & Female & Occupation & Male & Female \\
\hline \hline $18-25$ & 10 & 11 & Senior people & 4 & 2 \\
\hline $25-35$ & 8 & 9 & Students & 17 & 15 \\
\hline $35-45$ & 5 & 4 & Researchers & 5 & 4 \\
\hline $45-55$ & 4 & 3 & Engineers & 3 & 3 \\
\hline $55-60$ & 4 & 2 & Business people & 4 & 3 \\
\hline
\end{tabular}

tolerance of GeoPass was set to the same $21 \times 21$ pixel, while PassMap was set to $20 \times 20$ pixel.

Thus, our system can record users' inputs and construct a pattern like \{Sight, zoom level, the sequence of clicked places\}. The value of sight is either 0 (not selected) or 1 (selected). The initial zoom level is set to 2 and the maximum level is 18 . When users click on a map location, our system can record the relevant coordinate information. In order to further enhance users' memory, RouteMap uses a red arrow to mark the route, when users move mouse from one clicked place to the next location (see Figure 2).

\subsection{Evaluation}

To investigate the performance of RouteMap in the aspects of both long-term memory and multiple password inference, we conducted two user studies with 60 participants each.

\subsubsection{User Study with Common Participants}

This study involved 60 participants, among which 50 of them were from the former study in Section 3. The time gap between the two studies was one month. The newly attended participants also had no any background on information security. The detailed information of participants is described in Table 5.

In the study, we randomly divided 60 participants into two groups, named Groupl and Group2, and compared RouteMap with PassMap and GeoPass, respectively. More specifically, Group1 focused on RouteMap and PassMap, while Group2 focused on RouteMap and GeoPass. The implementation details of PassMap and GeoPass can refer to [38, 40]. Similarly, to avoid bias, we train all the participants based on the same steps on how to use these example systems.

Before the study, every participant has 3 trials to get familiar with the example systems. For example, participants in Groupl will create passwords for RouteMap and PassMap. All participants were guided and our study would not consider the data from these trials. In the study, we require all participants to create 5 passwords for each scheme in their group and each password corresponds to an account: the first password is created for an email account (personal use), the second one is created for a bank account, the third one is created for another email account (commercial use), the fourth one is created for a forum account and the last one is created for a social networking account. The detailed steps in each experiment are described as below:

- Experiment G1. Groupl conducted this experiment, in which each participant was required to firstly create 5 passwords for PassMap and then created 5 passwords for RouteMap after one hour rest.

- Experiment G2. Group2 conducted this experiment, in which each participant was required to firstly create 5 passwords for GeoPass and then created 5 passwords for RouteMap after one hour rest.

Both experiments followed the same steps, which are described as below:

- Step 1. Creation: creating a password following the related rules.

- Step 2. Confirmation: confirming the password by drawing the same secrets in the correct place. If users incorrectly confirm their password, they can retry the confirmation or return to Step 1.

- Step 3. Login: logging into the example system with all created passwords. Users can cancel an attempted login if they notice an error.

- Step 4. Feedback: All participants are required to complete a feedback form about the password creation and confirmation.

All participants had to finish the experiments in the same day. To compare the results with the previous study, after three weeks, we invited all participants to return and input all created passwords based on their own groups. Afterwards, we provided a feedback form to each participant regarding their password creation and memory.

Result analysis. The goal of this study is to investigate the multiple password memory of RouteMap by comparing it with similar schemes. The login success rates for Groupl and Group2 within three attempts are presented in Table 6. Our key observations are reported as below:

- Overall performance. As compared with the results in Table 2, it is found that participants performed much better in this study. Groupl could achieve a success rate of $75.3 \%$ and $88.7 \%$ for PassMap and RouteMap at the first attempt, respectively. After three attempts, the success rate could be increased to $85.3 \%$ and $93.3 \%$. On the other hand, Group 2 could achieve a success rate of $81.3 \%$ and $89.3 \%$ for GeoPass and RouteMap at the first attempt, respective1y. Then, the success rate can be elevated to $88.7 \%$ and 94\% after three attempts. 
Table 6: Login success rate for Group1 and Group2 after three weeks.

\begin{tabular}{|c|c|c|c|}
\hline Experiment G1 (PassMap) & Successful Login & Experiment G1 (RouteMap) & Successful Login \\
\hline 1st time & $113 / 150(75.3 \%)$ & 1st time & $133 / 150(88.7 \%)$ \\
\hline 2nd time & $125 / 150(83.3 \%)$ & 2nd time & $137 / 150(91.3 \%)$ \\
\hline 3rd time & $128 / 150(85.3 \%)$ & 3rd time & $140 / 150(93.3 \%)$ \\
\hline PassMap (Age in $[18,35])$ & Successful Login & RouteMap (Age in $[18,35])$ & Successful Login \\
\hline 1st time & $80 / 100(80.0 \%)$ & 1st time & $92 / 100(92.0 \%)$ \\
\hline 2nd time & $88 / 100(88.0 \%)$ & 2nd time & $94 / 100(94.0 \%)$ \\
\hline 3rd time & $89 / 100(89.0 \%)$ & 3rd time & $96 / 100(96.0 \%)$ \\
\hline PassMap (Age in $[35,45])$ & Successful Login & RouteMap (Age in $[35,45])$ & Successful Login \\
\hline 2nd time & $17 / 25(68.0 \%)$ & 2nd time & $20 / 25(80.0 \%)$ \\
\hline 2nd time & $19 / 25(76.0 \%)$ & 2nd time & $22 / 25(88.0 \%)$ \\
\hline 3rd time & $21 / 25(84.0 \%)$ & 3rd time & $22 / 25(88.0 \%)$ \\
\hline PassMap (Age in $[45,60])$ & Successful Login & RouteMap (Age in $[45,60])$ & Successful Login \\
\hline 1st time & $16 / 25(64.0 \%)$ & 1st time & $21 / 25(84.0 \%)$ \\
\hline 2nd time & $18 / 25(72.0 \%)$ & 2nd time & $21 / 25(84.0 \%)$ \\
\hline 3rd time & $18 / 25(72.0 \%)$ & 3rd time & $22 / 25(88.0 \%)$ \\
\hline Experiment G2 (GeoPass) & Successful Login & Experiment G2 (RouteMap) & Successful Login \\
\hline 1st time & $122 / 150(81.3 \%)$ & 1st time & $134 / 150(89.3 \%)$ \\
\hline 2nd time & $128 / 150(85.3 \%)$ & 2nd time & $136 / 150(90.7 \%)$ \\
\hline 3rd time & $133 / 150(88.7 \%)$ & 3rd time & $141 / 150(94.0 \%)$ \\
\hline "GeoPass (Age in $[18,35])$ & Successful Login & RouteMap (Age in $[18,35])$ & Successful Login \\
\hline 1st time & $80 / 90(88.9 \%)$ & 1st time & $84 / 90(93.3 \%)$ \\
\hline 2nd time & $86 / 90(95.6 \%)$ & 2nd time & $85 / 90(94.4 \%)$ \\
\hline 3rd time & $86 / 90(95.6 \%)$ & 3rd time & $87 / 90(96.7 \%)$ \\
\hline GeoPass (Age in $[35,45])$ & Successful Login & RouteMap (Age in $[35,45]$ ) & Successful Login \\
\hline 1st time & $14 / 20(70.0 \%)$ & 1st time & $17 / 20(85.0 \%)$ \\
\hline 2nd time & $14 / 20(70.0 \%)$ & 2nd time & $18 / 20(90.0 \%)$ \\
\hline 3rd time & $17 / 20(85.0 \%)$ & 3rd time & $18 / 20(90.0 \%)$ \\
\hline GeoPass (Age in $[45,60])$ & Successful Login & RouteMap (Age in $[45,60])$ & Successful Login \\
\hline 1st time & $28 / 40(70.0 \%)$ & 1st time & $33 / 40(82.5 \%)$ \\
\hline 2nd time & $28 / 40(70.0 \%)$ & 2nd time & $33 / 40(82.5 \%)$ \\
\hline 3rd time & $30 / 40(75.0 \%)$ & 3rd time & $36 / 40(90.0 \%)$ \\
\hline
\end{tabular}

- Age impact. It is easily imagine that the results for each age group would be improved, since the overall login success rate increased. Table 6 indicates that younger participants have advantages in memory while the success rate of senior people also increases a lot.

According to these observations, it is found that participants are able to better remember multiple passwords for these schemes, while our scheme can outperform the other two schemes, i.e., providing a higher success rate. The major reason is that RouteMap leads users to draw a route where they have experienced before. The experience actually enhances the relationship between different clicked places, so that users can have a better memory capability.

\subsubsection{User Study with FinTech Participants}

In this study, we recruited a total of 60 FinTech participants who have experience in a financial related job (e.g., accountant, financial service staff, financial manager), among which 20 of them were from the former study in Section 3. Table 7 describes the detailed information of FinTech participants.
Similar to the last study, we also randomly divided 60 participants into two groups: Group-A and Group-B. The participants in the first group focused on RouteMap and PassMap, and the participants in the second group focused on GeoPass and RouteMap. We trained all the participants based on the same steps on how to use these prototype systems, and each of them has 3 trials to get familiar with the example systems.

Similarly, we required all participants to generate 5 passwords for each scheme based on their group and each password corresponds to different accounts: a password for working desktop, a bank account, a corporate email account, a social networking account, and a personal email account, respectively. All participants followed the same steps in the above study, and completed the experiments in the same day. After three weeks, we invited all participants to return and validate all created passwords. Then, we provided a feedback form to them regarding the password creation and memory.

Result analysis. Table 8 describes the login success rate for each group within three attempts. It is found that the participants in Group- $A$ could achieve a success rate of $70.0 \%$ and 78.0\% for each PassMap and RouteMap at the first attempt. 
Table 7: Detailed information of participants in the user study with FinTech participants.

\begin{tabular}{|l|c|c|l|c|c|}
\hline Age Range & Male & Female & Occupation & Male & Female \\
\hline \hline $20-30$ & 15 & 13 & Accountant & 21 & 17 \\
\hline $31-40$ & 10 & 7 & Financial service staff & 8 & 9 \\
\hline $41-50$ & 7 & 8 & Financial manager & 3 & 2 \\
\hline
\end{tabular}

Table 8: Login success rate for Group-A and Group-B after three weeks.

\begin{tabular}{|l|l|l|l|}
\hline Group-A (PassMap) & Successful Login & Group-A (RouteMap) & Successful Login \\
\hline 1st time & $105 / 150(70.0 \%)$ & 1st time & $117 / 150(78.0 \%)$ \\
\hline 2nd time & $114 / 150(76.0 \%)$ & 2nd time & $126 / 150(84.0 \%)$ \\
\hline 3rd time & $120 / 150(80.0 \%)$ & 3rd time & $131 / 150(87.3 \%)$ \\
\hline \hline Group-B (GeoPass) & Successful Login & Group-B (RouteMap) & Successful Login \\
\hline 1st time & $112 / 150(74.6 \%)$ & 1st time & $115 / 150(76.7 \%)$ \\
\hline 2nd time & $122 / 150(81.3 \%)$ & 2nd time & $129 / 150(86.0 \%)$ \\
\hline 3rd time & $131 / 150(87.3 \%)$ & 3rd time & $135 / 150(90.0 \%)$ \\
\hline
\end{tabular}

With three attempts, the success rate can be finally changed to $80.0 \%$ and $87.3 \%$. For Group-B, the participants could reach a success rate of $74.6 \%$ and $76.7 \%$ for each GeoPass and RouteMap at the first attempt. With three attempts, they could increase the success rate to $87.3 \%$ and $90.0 \%$, respectively. For the age impact, it is very similar to the prior observation in Section 4.2.1 that younger participants have advantages in memory. As this observation is quite intuitive, it is not the main focus in this study.

As compared with the results in Table 6, we found there is no statistically significant difference for corresponding group. For example, the results have no statistically significant difference on PassMap and RouteMap $(\rho<0.05)$, between the participants from Groupl and Group-A. It is the same for GeoPass and RouteMap between the participants from Group2 and Group-B.

However, when we compare the results between PassMap and RouteMap, it is found a statistically significant difference. This is because PassMap requires users to select two locations on a map, which may increase the workload of remembering a password. By contrast, when we compare the results between GeoPass and RouteMap, it is interestingly found a statistically significant difference in Table 6 , whereas there is no statistically significant difference identified in Table 8. Based on our informal interview with most participants, the main reason should be the job background. As most participants in Table 6 have diverse background like students, researchers and Business people, the difference for each person may be more clear. Since most FinTech participants in Table 8 have a similar job background, it is not easy to identify such clear difference.

\subsubsection{Feedback}

To validate the observations, the major questions and relevant scores (feedback) are presented in Table 9. Ten-point Likert scales were used in each feedback question where 1-score indicates strong disagreement and 10-score indicates strong agreement.

- For the first three questions, it is found that most participants provided positive feedback for remembering these map-based passwords, in which our RouteMap received the highest score from both general and FinTech participants (with 9 and 8.8 respectively). Most participants reflected that the use of route in RouteMap could help improve remembering their created passwords, due to the correlation between these clicked places.

- For the following three questions, motivated by the usability, most participants were willing to use the map-based passwords in practice when they use email accounts and social networking accounts. In particular, RouteMap also received the highest score of 8.9 and 8.7 , respectively.

- Then, most participants showed a confidence in their created passwords. Among these map-based GP schemes, PassMap received the lowest score, as some participants concerned that friends might have a higher probability to guess their locations. In contrast, RouteMap obtained the best score, where most participants believed their passwords are hard to guess.

- For the last three questions, most participants believed that they could manage multiple GP passwords, in which RouteMap still received the highest score of 8.7 and 8.9 from both general and FinTech participants, as compared with the other two schemes (with an average score of 7.15 and 8.0 , respectively).

On the whole, according to the feedback, it is found that RouteMap could provide better authentication accuracy and multiple password memory for both general and FinTech participants.

\section{Further Discussion}

This work has investigated the issue of multiple graphical password inference for both general and FinTech users. In this part, we briefly discuss some issues of RouteMap, e.g., security and usability.

- Security aspect. As mentioned earlier, RouteMap is a kind of map-based password, which allows users to create a 
Table 9: Several main questions and relevant scores in the user study.

\begin{tabular}{|l|c|c|}
\hline Questions / Score (average) & General Participants & FinTech Participants \\
\hline \hline 1. I could easily remember PassMap passwords after one month & 7.3 & 6.9 \\
\hline 2. I could easily remember GeoPass passwords after one month & 8.1 & 8.2 \\
\hline 3. I could easily remember RouteMap passwords after one month & 9.0 & 8.8 \\
\hline \hline 4. Are you willing to use PassMap passwords in practice & 7.8 & 7.1 \\
\hline 5. Are you willing to use GeoPass passwords in practice & 8.5 & 8.3 \\
\hline 6. Are you willing to use RouteMap passwords in practice & 8.9 & 8.7 \\
\hline \hline 7. Do you think your PassMap is secure & 7.1 & 7.8 \\
\hline 8. Do you think your GeoPass is secure & 7.4 & 8.3 \\
\hline 9. Do you think your RouteMap is secure & 8.4 & 7.2 \\
\hline \hline 10. I can manage multiple PassMap passwords & 7.1 & 8.2 \\
\hline 11. I can manage multiple GeoPass passwords & 7.8 & 8.9 \\
\hline 12. I can manage multiple RouteMap passwords & 8.7 & \\
\hline
\end{tabular}

route as credentials through moving and clicking on several places on a world map. Intuitively, the password space would be larger than or at least equal to GeoPass (one clicked place on a map). However, due to the relationship between different clicked places, the password space may not keep increasing with more selected places. This is an interesting topic in our future work.

- Attacks on graphical passwords. Most graphical passwords are often vulnerable to various attacks, like 'hot spots' [2] and login-recording attacks [17]. In this work, we mainly focus on authentication accuracy and multiple password memory, it is one important topic to investigate the security of RouteMap under attacks.

- Usability aspect. Based on our results and participants' feedback, RouteMap could obtain higher scores than the other two schemes, we hence consider it can provide better usability. We also informally interviewed most general and FinTech participants, and most of them preferred RouteMap than the other two schemes. It is worth noting that the other two map-based schemes also obtain good feedback, when compared the scores between Table 3 and Table 9.

- Long-term password memory. Table 6 and Table 8 indicate that participants could generally have better long-term memory on map-based schemes, as compared with $D A S$ and PassPoints. Among the three map-based schemes, both general and FinTech participants could reach the best login success rate under RouteMap. The feedback in Table 9 also validated that participants believed that they could easily remember RouteMap passwords after one month.

- Multiple password memory. Our results in Table 6 and Table 8 demonstrate that users could achieve better memory of multiple passwords by using RouteMap than the other schemes. This is because users' memory can be enhanced by correlating the clicked places. The feedback in Table 9 also validated this observation, and most participants believed that they could handle multiple RouteMap passwords.

- Error tolerance. In the literature, GeoPass selected the error tolerance as $21 \times 21$ pixel, and PassMap used $20 \times 20$ pixel. Both studies have shown that the error tolerance over $20 \times 20$ pixel could achieve good usability. In this work, we adopted the error tolerance as $21 \times 21$ pixel.

- FinTech participants. In this work, we particularly recruited a total of 60 FinTech participants to help evaluate the inference of multiple graphical passwords on map-based schemes. As FinTech participants have a special focus on financial-related services, they sometimes are likely to show a more strict attitude towards an authentication system than general participants. This is because the intuitive security requirements in the FinTech domain. As most FinTech participants provided positive feedback on RouteMap, we believe the proposed scheme can be combined with the traditional textual passwords in practical financial applications. However, there is a need to tune RouteMap according to different security requirements.

\section{Conclusion}

Due to the popularity of FinTech applications, there is a need to design suitable authentication mechanisms to secure users' sensitive information. As traditional textual passwords suffer from many known issues, we focus on an alternative of graphical passwords in this work. First, we explored whether users could remember multiple graphical passwords for $D A S$ and PassPoints, and found that multiple password inference has become a big challenge. To address this issue, we introduce RouteMap, a map- and route-based graphical password scheme, in which users can create a route on a Google map as their credentials. To investigate its performance, we further conducted two user studies with 60 general participants and 60 FinTech participants, respectively. It is found that participants under RouteMap could achieve better long-term memory and multiple password memory, as compared with two similar schemes of PassMap and GeoPass. The feedback from both general and FinTech participants validated our observations. 
Our work aims to complement existing studies and stimulate more research of designing robust GP schemes in the area of FinTech. There are many promising topics in future, including providing a more specific analysis on password space and involving more participants with diverse background. Future work could also include conducting a thorough security analysis and evaluating map-based schemes in an adversarial environment (i.e., an attacker has some knowledge about the user and build a map password dictionary).

\section{Acknowledgment}

The authors would like to thank all participants for their hard work and cooperation in the user study. This work was partially supported by H2020 - CyberSec4Europe under the No. 830929.

\section{Reference}

[1] Bonneau, J.: The Science of Guessing: Analyzing an Anonymized Corpus of 70 Million Passwords. In: Proceedings of the 2012 IEEE Symposium on Security and Privacy, pp. 538-552 (2012)

[2] Chiasson, S., van Oorschot, P.C., Biddle, R.: Graphical Password Authentication Using Cued Click Points. In: Biskup, J., Lopez, J. (eds.) ESORICS 2007. LNCS, vol. 4734, pp. 359-374. Springer, Heidelberg (2007)

[3] Chiasson, S., Biddle, R., van Oorschot, P.C.: A Second Look at the Usability of Click-based Graphical Passwords. In: Proceedings of the 3rd Symposium on Usable Privacy and Security (SOUPS), pp. 1-12. ACM, New York (2007)

[4] Chiasson, S., Forget, A., Stobert, E., van Oorschot, P.C., Biddle, R.: Multiple password interference in text passwords and click-based graphical passwords. In: Proceedings of ACM Conference on Computer and Communications Security, pp. 500-511 (2009)

[5] Chiasson, S., Stobert, E., Forget, A., Biddle, R.: Persuasive Cued ClickPoints: Design, Implementation, and Evaluation of a Knowledge-Based Authentication Mechanism. IEEE Transactions on Dependable and Secure Computing 9(2), pp. 222-235 (2012)

[6] Davis, D., Monrose, F., Reiter, M.K.: On User Choice in Graphical Password Schemes. In: Proceedings of the 13th Conference on USENIX Security Symposium (SSYM), pp. 151-164. USENIX Association, Berkeley (2004)

[7] Dirik, A.E., Memon, N., Birget, J.C.: Modeling user choice in the passpoints graphical password scheme. In: Proceedings of the 3rd Symposium on Usable privacy and security (SOUPS), New York, NY, USA: ACM, 2007, pp. 20-28 (2007)

[8] Deloitte, FinTech in the CEE region, 2016. https: //www2.deloitte.com/content/dam/Deloitte/ global/Documents/About-Deloitte/central-europe/ ce-fintech-in-cee-region-2016.pdf

[9] P. Dunphy, J. Yan, Do background images improve "draw a secret" graphical passwords? In: Proceedings of the 14th ACM Conference on Computer and Communications Security (CCS), pp. 36-47 (2007)

[10] S. Fox. Future Online Password Could be a Map, 2010. http://www . livescience.com/8622-future-online-password-map.html.

[11] K. Gai, M. Qiu, X. Sun: A survey on FinTech. J. Network and Computer Applications 103, pp. 262-273 (2018)

[12] Georgakakis, E., Komninos, N., Douligeris, C.: NAVI: Novel Authentication with Visual Information. In: Proceedings of the 2012 IEEE Symposium on Computers and Communications (ISCC), pp. 588-595 (2012)

[13] Global FinTech Report 2017, https://www.pwc.com/ gx/en/industries/financial-services/assets/ pwc-global-fintech-report-2017.pdf

[14] Golofit, K.: Click passwords under investigation. In: Proceedings of the 12th European Symposium on Research in Computer Security (ESORICS). Berlin, Heidelberg: Springer-Verlag, pp. 343-358 (2007)

[15] Jermyn, I., Mayer, A., Monrose, F., Reiter, M.K., Rubin, A.D.: The Design and Analysis of Graphical Passwords. In: Proceedings of the 8th
Conference on USENIX Security Symposium, pp. 1-14. USENIX Association, Berkeley (1999)

[16] C.P. Katsini, C. Fidas, M. Belk, N.M. Avouris, G. Samaras: Influences of Users' Cognitive Strategies on Graphical Password Composition. CHI Extended Abstracts, pp. 2698-2705 (2017)

[17] W.-C. Ku, B.-R. Cheng, Y.-C. Yeh, C.-J. Chang: A Simple SectorBased Textual-Graphical Password Scheme with Resistance to LoginRecording Attacks. IEICE Transactions 99-D(2), pp. 529-532 (2016)

[18] D. Lin, P. Dunphy, P. Olivier, J. Yan. Graphical passwords \& qualitative spatial relations. In: Proceedings of the 3rd Symposium on Usable Privacy and Security (SOUPS), pp. 161-162 (2007)

[19] MacRae, B., Salehi-Abari, A., Thorpe, J.: An Exploration of Geographic Authentication Schemes. IEEE Transactions on Information Forensics and Security 11(9), pp. 1997-2012 (2016)

[20] Meng, Y.: Designing Click-Draw Based Graphical Password Scheme for Better Authentication. In: Proceedings of the 7th IEEE International Conference on Networking, Architecture, and Storage (NAS), pp. 39-48 (2012)

[21] Meng, Y., Li, W.: Evaluating the Effect of Tolerance on Click-Draw Based Graphical Password Scheme. In: Proceedings of the 14th International Conference on Information and Communications Security (ICICS), Lecture Notes in Computer Science 7618, Springer, pp. 349-356 (2012)

[22] Meng, Y., Li, W.: Evaluating the effect of user guidelines on creating click-draw based graphical passwords. In: Proceedings of the 2012 ACM Research in Applied Computation Symposium (RACS), pp. 322 327 (2012)

[23] Meng, Y., Li, W., Kwok, L.-F.: Enhancing Click-Draw based Graphical Passwords Using Multi-Touch on Mobile Phones. In: Proceedings of the 28th IFIP TC 11 International Information Security and Privacy Conference (IFIP SEC), IFIP Advances in Information and Communication Technology 405, pp. 55-68 (2013)

[24] Meng, W.: RouteMap: A Route and Map Based Graphical Password Scheme for Better Multiple Password Memory. In: Proceedings of the 9th International Conference on Network and System Security (NSS), pp. 147-161 (2015)

[25] Meng, W.: Evaluating the Effect of Multi-Touch Behaviours on Android Unlock Patterns. Information and Computer Security, vol. 24, no. 3, pp. 277-287, Emerald (2016)

[26] Meng, W., Li, W., Jiang, L., Meng, L.: On Multiple Password Interference of Touch Screen Patterns and Text Passwords. ACM Conference on Human Factors in Computing Systems (CHI), pp. 4818-4822 (2016)

[27] Meng, W., Li, W., Wong, D.S., Zhou, J.: TMGuard: A Touch Movement-based Security Mechanism for Screen Unlock Patterns on Smartphones. In: Proceedings of the 14th International Conference on Applied Cryptography and Network Security (ACNS), pp. 629-647 (2016)

[28] Meng, W., Li, W., Kwok, L.-F., Choo, K.-K.R.: Towards Enhancing Click-Draw Based Graphical Passwords Using Multi-Touch Behaviours on Smartphones. Computers \& Security, vol. 65, pp. 213-229 (2017)

[29] Meng, W., Li, W., Lee, W., Jiang, L., Zhou, J.: A Pilot Study of Multiple Password Interference between Text and Map-based Passwords. In: Proceedings of the 15th International Conference on Applied Cryptography and Network Security (ACNS), pp. 145-162 (2017)

[30] Meng, W., Liu, Z.: TMGMap: Designing Touch Movement-based Geographical Password Authentication on Smartphones. In: Proceedings of the 14th International Conference on Information Security Practice and Experience (ISPEC), pp. 373-390 (2018)

[31] Meng, W., Fei, F., Jiang, L., Liu, Z., Su, C., Han, J.: CPMap: Design of Click-Points Map-based Graphical Password Authentication. In: Proceedings of the 33rd IFIP TC-11 SEC 2018 International Conference on Information Security and Privacy Protection (IFIP SEC), pp. 18-32 (2018)

[32] Nelson, D.L., Reed, V.S., Walling, J.R.: Pictorial superiority effect. Journal of Experimental Psychology: Human Learning and Memory, vol. 2, no. 5, pp. 523-528 (1976)

[33] Passfaces, http://www.realuser.com/.

[34] Shepard, R.N.: Recognition memory for words, sentences, and pictures. Journal of Verbal Learning and Verbal Behavior, vol. 6, no. 1, pp. 156163 (1967)

[35] Shin, J., Kancharlapalli, S., Farcasin, M., Chan-Tin, E.: SmartPass: a s- 
marter geolocation-based authentication scheme. Security and Communication Networks 8, pp. 3927-3938 (2015)

[36] Spitzer, J., Singh, C., Schweitzer, D.: A Security Class Project in Graphical Passwords. Journal of Computing Sciences in Colleges 26(2), pp. 7-13 (2010)

[37] Suo, X., Zhu, Y., Owen, G.S.: Graphical Passwords: A Survey. In: Proceedings of the 21st Annual Computer Security Applications Conference (ACSAC), pp. 463-472. IEEE Computer Society, USA (2005)

[38] Sun, H., Chen, Y., Fang, C., Chang, S.: PassMap: A Map Based Graphical-Password Authentication System. In: Proceedings of ASIACCS, pp. 99-100, 2012

[39] Tao, H., Adams, C.: Pass-Go: A Proposal to Improve the Usability of Graphical Passwords. International Journal of Network Security 2(7), pp. 273-292 (2008)

[40] Thorpe, J., MacRae, B., Salehi-Abari, A.: Usability and Security Evaluation of GeoPass: a Geographic Location-Password Scheme. In: Proceedings of the 9th Symposium on Usable Privacy and Security (SOUPS), pp. 1-14 (2013)

[41] Vu, K.P.L., Proctor, R.W., Bhargav-Spantzel, A., Tai, B.-L., Cook, J., and Schultz, E.E. Improving Password Security and Memorability to Protect Personal and Organizational Information. International Journal of Human-Computer Studies, vol. 65, no. 8, pp. $744-757$ (2007)

[42] Wiedenbeck, S., Waters, J., Birget, J.-C., Brodskiy, A., Memon, N.: Passpoints: Design and Longitudinal Evaluation of A Graphical Password System. International Journal of Human-Computer Studies 63(12), 102-127 (2005)

[43] Weir, M., Aggarwal, S., Collins, M., Stern, H.: Testing Metrics for Password Creation Policies by Attacking Large Sets of Revealed Passwords. In: Proceedings of CCS, pp. 162-175 (2010)

[44] Yan, J., Blackwell, A., Anderson, R., Grant, A.: Password memorability and security: Empirical results. IEEE Security and Privacy, vol. 2, pp. 25-31 (2004)

[45] Yu, X., Wang, Z., Li, Y., Li, L., Zhu, W.T., Song, L.: EvoPass: Evolvable graphical password against shoulder-surfing attacks. Computers \& Security 70, pp. 179-198 (2017)

\section{Biography}

Weizhi Meng. is currently an assistant professor in the Cyber Security Section, Department of Applied Mathematics and Computer Science, Technical University of Denmark (DTU), Denmark. He obtained his Ph.D. degree in Computer Science from the City University of Hong Kong (CityU), Hong Kong. Prior to joining DTU, he worked as a research scientist in Infocomm Security (ICS) Department, Institute for Infocomm Research, A*Star, Singapore, and as a senior research associate in CS Department, CityU. He won the Outstanding Academic Performance Award during his doctoral study, and is a recipient of the Hong Kong Institution of Engineers (HKIE) Outstanding Paper Award for Young Engineers/Researchers in both 2014 and 2017. He is also a recipient of Best Paper Award from ISPEC 2018, and Best Student Paper Award from NSS 2016. His primary research interests are cyber security and intelligen$\mathrm{t}$ technology in security, including intrusion detection, smartphone security, biometric authentication, HCI security, trust computing, blockchain in security, and malware analysis. He served as program committee members for $20+$ international conferences. He is a co-PC chair for IEEE Blockchain 2018, IEEE ATC 2019, IFIPTM 2019, Socialsec 2019. He also served as guest editor for FGCS, JISA, Sensors, CAEE, IJDSN, SCN, WCMC, etc.

Liqiu Zhu. received his master degree in computer science from Macau University, Macau. He is currently a senior re- search engineer in a FinTech Startup in Macau, China. He has broad research interests including blockchain technology, artificial intelligent in security, smartphone security, IoT and Cyber Physical System (CPS) security.

Wenjuan $\mathrm{Li}$. is currently a Ph.D. student in the Department of Computer Science, City University of Hong Kong (CityU), and is holding an exchanged role at Technical University of Denmark (DTU), Denmark. Prior to this, she worked as a Research Assistant in CityU from 2013 to 2014, and was previously a Lecturer in the Department of Computer Science, Zhaoqing Foreign Language College, China. She was a Winner of Cyber Quiz and Computer Security Competition, Final Round of Kaspersky Lab Cyber Security for the Next Generation Conference in 2014. Her research interests include network management and security, collaborative intrusion detection, spam detection, trust computing, web technology and E-commerce technology. She is a student member of IEEE.

Jinguang Han. received his Ph.D from University of Wollongong, Australia, in 2013. He currently is a Lecturer in the Centre for Secure Information Technologies, Queens University Belfast, United Kingdom. His main research interests include access control, cloud computing, cryptography, and privacypreserving systems. He has served as a program co-chair of ProvSec 2016 and a program committee member of over 50 international conferences. He is a senior member of the IEEE.

Yan Li. is currently a Research Fellow at Singapore Management University. He received his Ph.D. degree in Information Systems from Singapore Management University in July 2014. His current research interests include privacy and security in social networks, face biometric-based user authentication, and liveness detection for face authentication. 